In our study we did not seek to determine whether the clinical care of individual patients was in any way affected by the CPA policy or whether the variation in original papers practice had a bearing on the quality of care given. Slavish adherence to guidelines does not necessarily guarantee quality of service (Marshall et al, 1997; Schneider et al, 1999). However, failure to fully apply clinical policies such as the CPA has lead to criticism of psychiatric services in a number of recent serious incident inquiries (Baroness Scotland of Asthal et al, 1998).

Health service policies are written to reduce variations and to eliminate unacceptable omissions in clinical practice. Where policies are developed in negotiation with clinicians, as was the case with the policy examined here, it is reasonable to expect closer adherence than was found in this study. It is possible that weaknesses in one aspect of a clinician's practice reflects problems elsewhere. Audits of routine matters such as the CPA may be one method of ensuring acceptable practice within the framework of clinical governance.

\section{References}

BINDMAN, J., BECK, A., GLOVER, G., et al (1999) Evaluating mental health policy in England. Care Programme Approach and Supervision registers. British Journal of Psychiatry, 175 327-330.
MACARTHY, A., ROY, D., HOLLOWAY, F. et al (1995) Supervision registers and the care programme approach: a practical solution. Psychiatric Bulletin, 19, 195-199.

MARSHALL, M. (1999) Modernising mental health services. British Medical Journal, 318, 3-4.

MARSHALL, M., GRAY, A. LOCKWOOD, A., et al (1997) Case management for severe mental disorders. In: The Cochrane Library. Oxford: Update Software.

PHILPOT, M. \& BANERJEE, S. (1997) Mental health services for older people in London. In London's Mental Health. The Report for the King's Fund London Commission (eds S. Johnson, R.

Ramsey, G. Thornicroft, et al). London: King's Fund Publishing.

—, SHEEHAN, B. \& REEVES, S. (1998) Use of the Care Programme Approach register by an inner-city old age psychiatry team. Psychiatric Bulletin, 22,772

* Michael Philpot Consultant, Mental Health for Older Adult Services, South London and Maudsley Hospital, Denmark Hill, London SE5 8AZ, tel: 08079192193 , fax: 020 79192961, e-mail: yum54@ dial.pipex.com, Heidi Hales Senior House Officer, Mental Health for OlderAdult Services, South London and Maudsley Hospital, Bart Sheehan Lecturer, Section of Old Age Psychiatry, Department of Psychiatry, TheWarneford Hospital, Oxford, Suzanne Reeves Honorary Specialist Registrar, Mental Health for Older Adult Services, South London and Maudsley Hospital, Martin Lawlor CPA Administrator, South London and Maudsley Hospital

\title{
Use of a prescribing protocol in routine clinical practice: experience following the introduction of donepezil
}

\author{
AIMS AND METHOD \\ Following the introduction of done- \\ pezil into clinical practice a protocol \\ for prescribing it was developed in \\ Leicestershire. A prospective clinical \\ audit was undertaken to monitor \\ compliance with the protocol, which \\ also provided an opportunity to \\ evaluate the outcome of therapy in \\ routine clinical practice.
}

\author{
RESULTS \\ Overall there was close adherence to \\ the protocol by the clinicians and \\ clinical factors, as well as organisa- \\ tional and resource-related factors, \\ were important in determining who \\ received treatment. The principal \\ outcome measures (Mini-Mental \\ State Examination, Barthel ADL Index \\ and Clinical Dementia Rating Scale)
}

did not demonstrate any significant treatment effect.

\section{CLINICAL IMPLICATIONS}

This study demonstrates the feasibility and acceptability of using a protocol-based approach to manage the introduction of new drug treatments in psychiatry.
Donepezil was the first drug to receive a licence in the UK (in the spring of 1997) for the treatment of Alzheimer's disease. At the time there was only one published clinical trial using the drug (Rogers \& Friedhoff, 1996) and this had shown only modest improvement in cognitive performance in patients with Alzheimer's disease. It was estimated that the annual cost of drug treatment (excluding any other costs, e.g. investigations) would be about $f 1000$ per patient. As a result there was widespread uncertainty among both clinicians and health service managers in many parts of the country concerning the cost-effectiveness of providing this treatment (Alzheimer's Disease Society, 1997). Thus, in some parts of the country the policy was simply not to prescribe, while in other areas local guidelines were developed for prescribing donepezil (Harvey, 1999).

In Leicestershire it was decided that donepezil should be available but the treatment should be targeted at patients for whom there is evidence of 
benefit. To achieve this a local protocol for prescribing cholinesterase inhibitors was developed and employed from the outset (see Appendix). One of the requirements of the protocol was that the pattern of use of donepezil and compliance with the protocol should be monitored. As a consequence a prospective clinical audit was undertaken. The audit also provided an opportunity to examine systematically the outcome of therapy in a routine clinical setting, an area in which there are few published data at present.

\section{Method}

\section{Setting}

The audit was carried out at the Bennion Centre, Glenfield Hospital, Leicester. This is the base hospital for oldage psychiatric services covering the western half of the city of Leicester and Leicestershire, with a catchment population of approximately 70000 persons aged 65 and over.

\section{Subjects and procedures}

All patients initially considered for donepezil treatment between 1 August 1997 and 31 July 1998 were included in the audit. They received a standardised assessment of dementia severity, cognitive and functional status and other parameters. This included the Clinical Dementia Rating scale (CDR; Hughes et al, 1982), Mini-Mental State Examination (MMSE; Folstein et al, 1975), Barthel ADL (activities of daily living) Index (BAl; Mahoney \& Barthel, 1964) and a check-list covering various non-cognitive features of dementia. Case notes were reviewed to determine the basis for the diagnosis of probable Alzheimer's disease. Following the initial assessment those who then went on to receive the treatment were reassessed 8-12 weeks later with the same measures. In addition, pre- and post-treatment questionnaires were sent to each patient's main carer to assess their knowledge and expectations of treatment.

\section{Results}

\section{Patient selection and characteristics}

Between 1 August 1997 and 31 July 1998, 62 patients were considered for donepezil treatment. Of these, 35 eventually received treatment. The characteristics of the treated and untreated groups are shown in Table 1. The groups differed only in mean age $(t=3.52, P=0.001)$. Reasons for eventual non-treatment included failure to meet diagnostic or other eligibility criteria (44\%), refusal of the general practitioner to continue treatment (30\%) and other factors including problems of follow-up (26\%); for example, patient moving out of the area. Of the 35 treated patients, complete 12-week follow-up data were available for 25 of them. Four patients had discontinued before 12 weeks and for the remainder only incomplete data were available.
With respect to non-cognitive symptoms there were no significant differences between the treated and nontreated groups with the exception of weight loss and aggression, which were present in a significantly greater number of non-treated $(P<0.05$; Fisher's exact test).

\section{Therapeutic outcome}

Mean changes in MMSE, BAI and CDR-SB (sum of boxes) scores were not statistically significant (see Table 2) although a few individuals showed larger changes on one or both measures (see Fig. 1). These findings were broadly in line with clinicians' general impressions of a small minority of patients demonstrating a marked overall clinical change.

Sixty-nine per cent of carers reported 'some' or 'marked' improvement (number of responders $=16$ ). The most frequently cited areas of improvement were in $\operatorname{mood}(n=7 ; 44 \%)$ and sociability $(n=8 ; 50 \%)$. In eight cases $(50 \%)$ carers stated that the degree of improvement was less than they had anticipated.

\section{Discontinuation of treatment}

At the 12-week follow-up four of the 35 patients discontinued treatment; two owing to side-effects (gastrointestinal), one owing to rapid deterioration and one owing to death (unrelated to treatment). In addition to this, five carers reported possible side-effects that were mild and transient. These included minor gastrointestinal upset (two), insomnia (one), mood swings (one)

\section{Table 1. Characteristics of treated and non-treated patients}

\begin{tabular}{|lcc}
\hline & $\begin{array}{c}\text { Treated } \\
(n=35)\end{array}$ & $\begin{array}{c}\text { Non-treated } \\
(n=27)\end{array}$ \\
\hline Mean age & $71.8^{* * *}$ & 79.2 \\
Male:female & $13: 22$ & $7: 20$ \\
Mean MMSE score & 20.4 & 18.9 \\
Mean BAl score & 18.9 & 17.7 \\
Severe dementia - CDR $>2(\%)$ & $1(2.9)$ & $3(11.1)$ \\
Living with spouse or family (\%) & $24(68.6)$ & $12(44.4)$ \\
\hline
\end{tabular}

$* * * t=3.48 ; P=0.001$.

MMSE, Mini-Mental State Examination; BAI, Barthal ADL (activities of daily living) Index; $C D R$, Clinical Dementia Rating scale.

Table 2. Mean Mini-Mental State Examination (MMSE), Barthal ADL (activities of daily living) Index (BAI) and Clinical Dementia Rating scale - sum of boxes (CDR-SB) scores: baseline and post-treatment $(n=25)$

\begin{tabular}{|lcccc} 
& Mean & $\begin{array}{c}\text { Mean } \\
\text { post- } \\
\text { baseline } \\
\text { treatment }\end{array}$ & $\begin{array}{c}\text { Mean difference } \\
(95 \% \mathrm{Cl})\end{array}$ & P value \\
\hline MMSE & 21.0 & 21.9 & $0.9(-0.1$ to 1.9$)$ & 0.09 \\
BAl & 18.6 & 18.9 & $0.3(-0.3$ to 0.8$)$ & 0.11 \\
CDR-SB & 6.2 & 6.0 & $-0.2(-1.1$ to 0.7$)$ & 0.39 \\
& & & & \\
\hline
\end{tabular}

original papers 


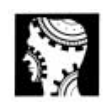

original papers

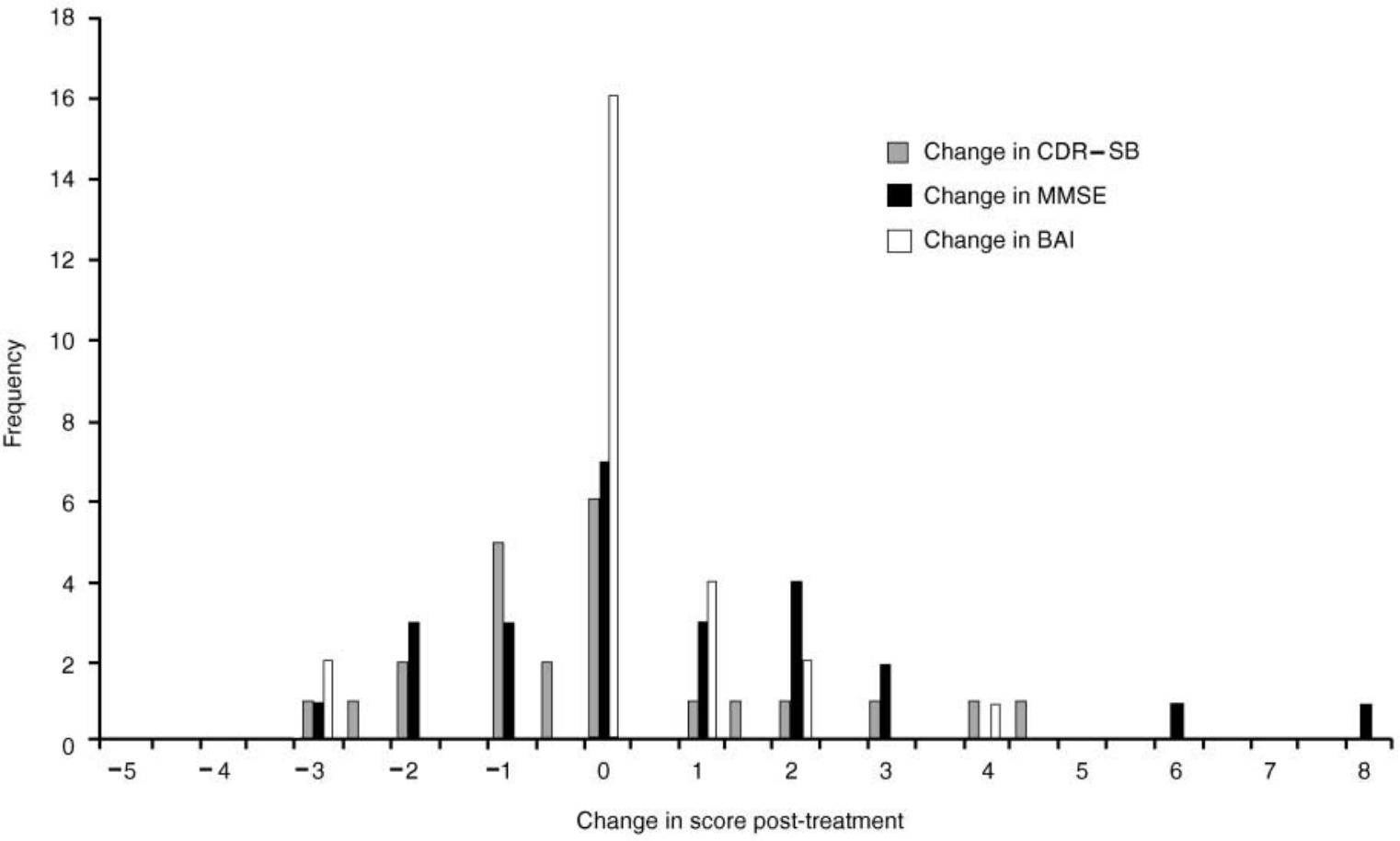

Fig. 1 Change in Clinical Dementia Rating scale - sum of boxes (CDR-SB), Mini-Mental State Examination (MMSE) and Barthel ADL (activities of daily living) Index (BAI): post-treatment against baseline

and muscular aches (one). As at 31 July 1999, a further 22 patients had discontinued treatment; nine owing to lack of benefit, 12 owing to deterioration and one owing to death (again, this was unrelated to treatment). There were no further cases of discontinuation because of sideeffects and nine of the 35 patients who had commenced treatment were still continuing treatment as at 31 July 1999. Of the 26 patients who had discontinued treatment, $50 \%$ had discontinued by 30 weeks and approximately two-thirds (65\%) by 12 months.

\section{Conclusions}

The characteristics of the patients selected for treatment with donepezil were broadly in line with the eligibility criteria contained in the protocol. Acceptance of the protocol by the clinicians did not present any significant problems despite the additional work involved in administering the rating scales and questionnaires. During the period described, it appears that resource-related and organisational factors were as important as clinical factors in determining who received treatment. The total number of patients was far less than had been originally anticipated. Although the annual cost of prescribing donepezil has been estimated at $£ 1000$ per patient, a significant proportion of the patients did not complete 1 full year's treatment. Thus, the average drug cost per patient treated was significantly less than the estimated cost.
Generally, donepezil appears to be well tolerated, with only two of the 35 patients in this series discontinuing treatment because of adverse effects. The objective outcome measures employed in this evaluation did not demonstrate a large treatment effect. This would be consistent with either a genuine lack of clinical benefit; with a relative insensitivity of these measures in the target domains together with the possibility of type two error (owing to small numbers); or with a failure to rate those domains that may, in fact, manifest the greatest response to treatment. The latter possibility is reinforced by the subjective clinician and carer impressions of improvement in mood and social functioning. Other authors have also reported noticeable improvements in the non-cognitive features of the condition and the relief and enhancement in quality of life experienced by the patients' carers (Burns et al, 1999; Watts-Tobin \& Horn, 1999). It will be important in future to incorporate into anti-dementia drug trials and evaluations valid measures of personal functioning that assess those areas in which carers report worthwhile improvement.

Finally, donepezil was the first drug (apart from clozapine) prescribed by psychiatrists in Leicestershire to be subjected to a clearly defined protocol for its use. Overall, we did not encounter any significant problems in incorporating the protocol into routine clinical practice. The systematic collection of data on clinical experience with newly introduced drugs usefully complements the evidence available from clinical trials: our experience indicates the feasibility of this approach and its acceptability to prescribers. 


\section{References}

ALZHEIMER'S DISEASE SOCIETY (1997) Newsletter. November 1997. London: Alzheimer's Disease Society.

BURNS, A. RUSSELL, E. \& PAGE, S. (1999) New drugs for Alzheimer's disease. British Journal of Psychiatry, 174, 476-479.

FOLSTEIN, M. F., FOLSTEIN, S. E. \& McHUGH, P. R. (1975) "Mini-mental state": a practical method for grading the cognitive state of patients for the clinician. Journal of Psychiatric Research, 12, 189-198.

HARVEY, R. (1999) A review and commentary on a sample of 15 UK guidelines for the drug treatment of Alzheimer's disease. International Journal of Geriatric Psychiatry, 14, 249-256.
HUGHES, C. P., BERG, L., DANZIGER, W. L., et al (1982) A new clinical scale for the staging of dementia. British Journal of Psychiatry, 140, 566-572.

MAHONEY, F. I. \& BARTHEL, D.W.

(1964) Functional evaluation: the Barthel index. Maryland State Medical Journal, 14, 61-65.

ROGERS, S. L. \& FRIEDHOFF, L.T. (1996) The efficacy and safety of donepezil in patients with Alzheimer's disease: results of a US multicentre, randomized, double blind, placebo-controlled trial. Dementia, 7, 293-303.

WATTS-TOBIN, M. A. \& HORN, N. (1999) Prescribing donepezil in clinica practice. British Journal of Psychiatry, 175, 393.

\section{Acknowledgement}

This project was supported in part by an unrestricted grant from Pfizer Ltd.

*Richard Prettyman Senior Lecturer, Jaiker Jari Consultant, University of Leicester, Division of Psychiatry for the Elderly, the Bennion Centre, Glenfield General Hospital, Groby Road, Leicester LE3 9DZ

\section{Appendix}

The Leicestershire Mental Health Service NHS Trust Protocol for the prescribing of cholinesterase inhibitor drugs: 1997 Version ${ }^{1}$

original papers

(a) Patients should have a diagnosis of probable Alzheimer's disease. Although this should be a clinical judgement, National Institute of Neurological and Communicative Disorders and Stroke/Alzheimer's Disease and Related Disorders Association criteria are suggested as guidelines for making the diagnosis.

(b) Disease should not have progressed to the severe stage. The Cambridge Examination for Mental Disorders of the Elderly severity classification is suggested as a model for assessing the disease stage.

(c) There should be assurance of supervision when administering medication in the domestic setting

(d) Treatment is to be initiated by the specialist but the responsibility of ongoing prescribing to be that of the patient's general practitioner.

(e) Treatment should be discontinued if disease progresses to the severe stage or if there is no benefit from the treatment after an adequate trial.

(f) The treatment response should be regularly monitored - initially at 2-4 weeks, then at 12 weeks and then at 12 -weekly intervals.

1. The protocol currently followed in Leicestershire is a modified version of the 1997 protocol but the principal components are essentially the same.

\section{RAHUL RAO}

\section{'Sadly confused': the detection of depression and dementia on medical wards}

\section{AIMS AND METHOD}

Dementia and depression are common psychiatric diagnoses in older people, and are common reasons for referral to liaison psychiatry services. The present study examined the accuracy of physicians' diagnoses for both disorders in consecutive referrals to a liaison old age psychiatry service.

\section{RESULTS}

Positive predictive values for depression and dementia were high, but levels of treatment of depression and documentation of past psychiatric history were both poor. Alcohol misuse and stroke accounted for the commonest accompanying disorders.

\section{CLINICAL IMPLICATIONS}

The findings have implications for the encouragement of physicians to treat depression when this is suspected. Educational programmes for this purpose may be useful, incorporating an exploration of attitudes and knowledge of physicians towards depression in older people.
The prevalence of psychiatric disorders in older people with acute physical illness is high, with depression known to be present in over 30\% (Sadavoy et al, 1990; Evans, 1993) and dementia in over $14 \%$ (Feldman et al, 1987; Turrina et al, 1992) of elderly in-patients.

Recognition of both depression and cognitive impairment is vital, having implications for treatment, social/carer support and prognosis. Most studies examining the recognition of depression have been confined to primary care settings, with some evidence that the detection of depression by general practitioners may be more adequate than both treatment and referral to specialist services (MacDonald, 1987).

The possibility of either low mood or cognitive impairment may influence referrals to liaison psychiatry services, but the appropriateness of such referrals and accuracy of suspected diagnoses deserves further exploration. 